\title{
Liver hydatid cyst: Pattern and methods of surgical treatment drainage versus omentoplasty
}

\author{
Omar Eissa, ${ }^{a} M D$; Mohamed Rady, ${ }^{b} M D ;$ Nader Kotb, ${ }^{b} M D$; \\ Ans Meshal, ${ }^{b} M D$; Ahmed Gamal EL-Din, ${ }^{b} M D$
}

\author{
a) Department of General Surgery, Menia University, Menia, Egypt. \\ b) Department of General Surgery, Ain Shams University, Cairo, Egypt.
}

\begin{abstract}
Background: The surgical treatment technique for liver hydatic cyst (LHC) cannot be standardized, and the surgical technique should be tailored according to the extent of the cyst and any adjunct complications of hydatid disease. Liver is the most common site of infection and several methods of surgery have been described to treat this common disease. In this study we aimed to compare the results of two common methods of surgery;simple drainage versus omentoplasty.

Patients and methods: In this prospective study 25 patients with hepatic hydatid cyst underwent surgery from January 2008 to January 2013. 15 patients were treated with omentoplasty (group I) and the other ten patients were treated with simple tube drainage (group II). The results of surgery including mortality, complications and recurrences were recorded.

Results: There was no case of mortality in each group of patients. Postoperative complications were seen in $6.7 \%$ of group I, 15\% of group II patients. The mean duration of hospital stay was 4.5 and 8.6 days in group I and group II patients. During a mean period of 18.6 month follow up there was no recurrence in each group of patients.

Conclusion: According to the results of this study we suppose that omentoplasty of cyst cavity -if feasible-is preferred to tube drainage.
\end{abstract}

Key words: Drainage, hydatid cyst, omentoplasty.

\section{Introduction:}

Hydatid disease has been known since the time of Hippocrates and is described as a 'liver full of water' ${ }^{1}$ Hydatid disease is a zoonosis caused mainly by Echinococcus granulosus, or less frequently by Echinococcus multilocularis and Echinococcus oligarthrus. The primary carriers are dogs and wolves. The intermediate hosts are sheep, cattle, and deer. Humans are alternative / accidental secondary hosts and are infected by ingestion of ova from the feces of dogs. ${ }^{2}$

Endemic regions of human cystic disease include South America, the Mediterranean region including North Africa, Spain,
Protugal, Turkey, the Middle East, central Asia and many regions in China. ${ }^{3}$ The parasite "Echinococcus granulosus" is a cestode that grows in the small intestine of its definite host usually a dog. The host puts off eggs of the parasite within stools and when an intermediate host (sheep or human) ingest vegetables contaminated with definite host feces, larvae of the parasite exit the eggs in the duodenum. The larvae pass through the intestinal wall and reach the liver via the portal system where they form cysts.

In humans $50 \%$ to $75 \%$ of hydatid cysts occur in the liver, $25 \%$ are found in the lungs and $5 \%$ to $10 \%$ are distributed to other tissues 
along the arterial system. ${ }^{4}$

Without treatment, cysts grow and eventually form fistulas into adjacent organs or rupture into the peritoneal cavity. Older cysts have an increased risk of daughter cyst formation, which is an important factor for recurrence of disease after surgery. ${ }^{5}$

Several types of treatment have been described to treat hydatid cyst of the liver. Medical therapy alone is insufficient to cure the disease, although stabilization of disease has been reported with albendazole alone or in combination with praziquantel. Surgical approaches vary from complete resection (e.g. total pericystectomy or hepatectomy) to minimal invasive procedures (e.g. percutaneous aspiration of cysts).

Complictions are observed in one third of patients with liver hydatid cysts. ${ }^{6}$ Anaphylactic shock, cyst infection of the biliary tree, and rupture into the peritomeum are the most common complications. Intrabiliary rupture is reported to be seen in a range of $6.1-17 \% .^{7}$

Symptoms are created by the compression or displacement of other structures or organs, with subsequent jaundice, adverse effects on hollow organ motility, and exceptionally spontaneous or traumatic rupture. ${ }^{8}$

The choice of therapy depends on several factors: general condition of the patient, number and localization of the cysts, the surgeon's experience and the presence of special services such as intensive care unit. This study presents the long- term results of two surgical techniques in treating hepatic hydatid cyst and compares their postoperative complications, morbidity and recurrence of the disease.

Most patients with a hydatid cyst in the liver have no symptoms, and its presence becomes evident when the liver is found to be enlarged or a cystic lesion is noted when the liver is imaged for other reasons. Large cysts may be painful, but otherwise symptoms may be the result of a number of complications. They may rupture into the biliary system and then the patient presents with cholangitis. Cysts may also become infected or obstruct major intrahepatic bile ducts. Subcapsular cysts may rupture into the peritoneal cavity and the patient may experience anaphylactic shock. ${ }^{9}$

\section{Patients and methods:}

Twinty-five patients, who underwent surgery for hepatic hydatid cysts between January 2009 and December 2013, were included in this study. Patients were divided into two groups. The first group consisted of 15 patients treated with omentoplasty and the second group were 10 patients treated surgically with tube drainage of the cyst cavity. In all cases abdominal ultrasonography and abdominal CT were done. Hepatic infestation with Echinococcus granulosus was confirmed histologically in all patients. Surgical techniques were compared with respect to post-operative complications, hospital stay and recurrence of the disease. Patients were invited to be visited every 3 months, at which clinical examination, ultrasonography and serological tests were performed.

\section{Surgical techniques:}

In both techniques we used an extended right subcostal incision. After entering the abdominal cavity, routine exploration of abdominal viscera was done as well as the liver and the cyst. The surgical field was covered with packs immersed in scolicidal agent (silver nitrate $0.5 \%$ or hypertonic saline $20 \%$ ) to prevent the spread of the parasite and reduce the risk of intraperitoneal soiling and contamination.

Then the cyst was evacuated by aspiration with a closed system suction device. After complete aspiration of cyst contents, the cyst was unroofed and the germinal layer and the remaining daughter cysts were removed. Then we put clean sponges over the inner layer of the cyst to define bile leakage from biliary openings. If there was any biliary opening, each one was ligated to prevent postoperative bile leakage. Then the cyst cavity was filled with silver nitrate immersed sponges for a few minutes to sterilize the cavity of the cyst and then sponges were removed. At this point, the first group of patients were considered to fill the cavity of their cysts with omentum and the second group were treated with simple tube 
drainage (closed suction) of the cyst cavity.

\section{Results:}

25 patients (16 male and 9 female) were included in this study with a mean age of 31.8 years (rang 14-65 years). 22(88\%) cysts were located in the right lobe and three $(12 \%)$ in the left lobe of the liver. The mean diameter of the cysts was $12 \mathrm{~cm}$ (rang 6-25cm). Postoperative complications including atelectasia, wound infection and abscess formation were compared in two groups of patients. Atelectasia as a common complication of abdominal surgery was common in both groups of patients with no significant difference. Wound infection was seen in two patients $(20 \%)$ of group II and one patient $(6.7 \%)$ of group I. Intraabdominal abscess formation occurred in one (10\%) patient of group II. Overall complication rate in group I patients was $(6.7 \%)$ and in group II patients it was $15 \%(\mathrm{p}<0.05)$.

The mean postoperative hospital stay was significantly longer after drainage procedures (8.6 days) than those treated with omentoplasty $(4.5$ days $)(\mathrm{p}<0.05)$. In a mean period of 18.6 (range 13-29) month of follow up there was no case of recurrence based on physical serologic tests in each group of patients.

\section{Disscusion:}

The natural history of echinococcosis is insidious and usually asymptomatic till reaching a large size stretching Glissons (liver) capsule or complicated by infection or hemorrhage, hence high index of suscpision is necessary to diagnose and prevent life threatening complications and morbidity. If the cyst ruptures, scolices can grow in the peritoneum, pleura, bronchial tree, and bile ducts. ${ }^{10}$ Liver hydatidosis is a common problem, associated with chronic morbidity and has variable clinical manifestations, for these reasons it should be included in differential diagnosis of any abdominal pain, mass or acute abdomen. ${ }^{11}$

The majority of patients have single-organ involvement $(87 \%)$ and a solitary cyst (72\%) at the right lobe of the liver. These findings are nearly the same as in our study results where we have $(88 \%)$ a single organ involvement and $(80 \%)$ solitary cyst located in the right lobe of the liver. The right lobe of the liver is more affected in our study and other similar studies due to its largest blood supply. ${ }^{12}$

In contrast to western countries where the disease is incidental finding, in middle east and our study the commonest presenting symptoms is upper abdominal pain and/ or discomfort, which reflect a delay in medical consultation among our patients and under assessment of patients clinically and radiologically. It is known that $(6 \%)$ of acute abdomen is due to interaperitoneal rupture of liver hydatid cysts. ${ }^{13}$

Different surgical techniques and procedures have been carried out and even in some cases, a liver transplant has been required. Advances in drug therapy has been influenced by the introduction of albendazole and accelerated by addition of praziquantel, but this requires a long period of treatment i.e. up to a year or more, and is not effective for everyone.

Surgical treatment still remains the treatment of choice in the management of hydatid disease. However, supplementary medical therapy may be necessary to prevent recurrence in high risk group. ${ }^{12}$

The principles of hydatid surgery include removal of all infective cyst parts; inactivation of the cyst cavity, avoidance of intra-abdominal spillage of the cyst contents, elimination of all viable elements (endo cyst with its contents; scolices, fluid and daughter cysts) and management of the residual cavity. ${ }^{14}$

Two main operative approaches have been described in this study; (1) drainage procedure, (2) the obliteration of the cavity after evacuation of the cystic contents. Many debatable results have been reported in relation to the surgical treatment of cystic cavity. The procedures performed depend on the location and characteristics of the cysts and on the general condition of the patient. Traditionally, various surgical alternatives have been described such as pericystectomy, cystectomy (partial or complete) with 
external drainage, marsupilisation, capiitonage or omentoplasty. 15 Particularly, these procedures are not always applicable and a few of them have been abandoned nowadays. In contrast, the laparoscopic approach is rapidly developing following the progress of modern laparoscopic equipments (e.g perforator grinder, aspirator apparatus and locking umbrella trochar). ${ }^{16}$ The reported advantages of laparoscopic approach are minimal invasiveness, shorter hospital stay and reduced wound complications. Disadvantages of such approach include the limited manipulation area, potentional risk of spillage during puncture and the high risk of bleeding in centrally located cyst. ${ }^{17}$ Recently, laparoscopy is quite feasible to perform in hydatid disease of the liver, and the use of helical fasteners allows effective omental flap fixation. ${ }^{18}$

Pericystectomy may be hazardous or even impossible to perform and is usually limited to small, peripheral cysts of anterior right lobe or left lobes. ${ }^{19}$ Such operations have high morbidity and mortality rates and can be considered radical procedures for such a bengin disease. Hepatic resection should only be considered for Echinococcus alveolaris cases that are located on one hepatic lobe.

Total pericystectomy is usually restricted to small, peripherally located liver cysts that are only partially surrounded by parenchyma. ${ }^{20}$

Cystectomy and omentoplasty is safe, simple and effective where the omentum acts as a biological drain and natural sealing agent. ${ }^{21}$

Drainage procedures can be followed by various post-oprative complications, such as hepatic abscess, biliary fistula, and a longer hospital stay. Therefore omentoplasty for a single uncomplicated hydatid cyst caused significantly fewer complications than external drainage, and reduced hospital stay. ${ }^{22}$ However, in our experience in the group treated by drainage of the cystic cavity increased there was post-oprative morbidity in comparison with the other group treated by omentoplasty. The difference was statistically significant $(\mathrm{P}>0.05)$. The restriction of activity in patients with tube drainage was also an important predisposing factor for pulmonary infection and thromboembolism. Furthermore, The drain was an important factor in the enterance of microorganisms into the peritoneum thus increasing peritoneal infections. However, external tube drainage retains its value as a simple and safe procedure. ${ }^{23}$

Omentoplasty seems to be the best possible surgical alternative for the radical treatment of hepatic hydatid cysts. The management of liver hydatid cysts should be flexible, taking into consideration a number of factors and variables. ${ }^{15}$

Omentoplasty should be the standard surgical procedure because it is safe, simple and effectively meets all criteria of surgical treatment for hydatid disease: entire elimination of the parasite, no intra operative spillage, and saving healthy tissues. ${ }^{24}$

Therfore no single method can be recommended for the treatment of hepatic hydatid cysts but the choice of the surgical methods must be made according to the complications of the cyst. Omentoplasty is the procedure of choice for uncomplicated cysts with low complication rate and relatively short hospital stay. External tube drainage is recommended for infected cysts and a biliary drainage procedure should be added to external tube drainage for cysts with intrabiliary rupture.

\section{Conclusion:}

As many other authors, we conclude from this study, that excision of the cyst and omentoplasty is the procedure of choice; having the lowest morbidity rate. Thus surgical procedures do not employ drainage as the treatment of choice in the management of patients with hydatid cyst of the liver External drainage could occasionally be reserved for more complicated cases and where there is sepsis of the biliary tree.

\section{References:}

1- Sayek I, Yalin R, Sanac Y: Surgical treatment of hydatid disease of the liver. Arch Surg 1980; 115: 847-850.

2- Harris KM, Morris DL, Tudor R, Toghill 
P, Hardcastle JD: Clinical and radiographic features of simple and hydatid cysts of the liver. Br J Surg 1986; 73: 835-838.

3- Sparchez Z, Osian G, et al: Ruptured hydatid cyst of the liver with biliary bstruction:presentation of a case and review of the literature. Rom J Gastroentrol 2004; 13: 245-250.

4- Attef M Elshazly, Manar S Azab, et al: Hepatic hydatid disease: Four case reports. Cases J 2009; 2: 58.

5- Magistrelli P, Coppola R, Messia A, et al: Surgical treatment of hydatid disease of the liver; A 20 years experience. Arch Surg 1991; 126: 518-522.

6- Akcan A, Sozuer E, Akyildiz H, et al: Prediposing factors and surgical outcome of comlicated liver hydatid cysts. World $J$ Gastroeterol 2010; (24): 3040-3048.

7- Serhat C, Unal Z, Arsla H: Intrabiliary rupture of liver hydatid cyst: A case report and review of the litrature Cases $J$ 2009; 2: 6455.

8- Placer C, Martin R, Sanchez E, Soleto E: Rupture of abdominal hydatid cysts. $\mathrm{Br} \mathrm{J}$ Surg 1988; 75: 157.

9- Zhang W, Li J, McManus DP: Concepts in immunology and diagnosis of hydatid disease. Clin Microbiol Rev 2003; 16: 18-36.

10-Elton C, Lewis M, Jourdan MH: Unusual site of hydatid disease. Lancet 2000; 355: 2132.

11-Filippou D, Tselepi D, Filippou G, Papadopoulos V: Advances in liver echinococcosis: Diagnosis and treatment. Clin Gastroenterol Hepatol 2007; 5: 152-159.

12-Jenkis DJ, Roming T, Thompson RC: Emergency/re-emergency of echinococcus spp. a global update. Int J Paracytol 2005; 35: 1205-1219.

13-Sotirak S, Himonas C, Korkoliokou P: Hydatosis - ecinococcosis in Greece. Act Trop 2003; 85: 197-201.

14-Akugan Y, Yilmaz G: Efficiency of obliteration procedures in the surgical treatment of hydatid disease of the liver. ANZ J Surg 2004; 74: 968-973.

15-Utkan NZ, Canturk NZ,Gonullu N,Yildrir C, Dulger M: Surgical experience of hydatid disease of the liver; omentoplasty or capiitonage versus tube drainage. Hepatogastroenterology 2001; 48(37): 203-207.

16-Milicevic M, Bumgart LH, Fong Y: Hydatid disease. In: Surgery of the liver and biliary tract. Philadelphia: Saunders (Publisher); 2000; p.1167-1204.

17-Sayek I, Onat D: Diagnosis and treatment of uncomplicated hydatid cyst of the liver. World J Surg 2001; 25: 21-27.

18-Altinli E, Saribeyoglu K, Pekmzci S, et al: An effective omentoplasty technique in laparoscopic surgery for hydatid disease of the liver. JSLS 2002; 6(4): 323-326.(h31).

19-Pelaez V, Kugler C, Correa D, et al: Pair as percutaneous treatment of hydatid liver cysts. Acta Trop 2000; 75: 197-202.

20-Ayles HM, Corbet EL, Taylor I, et al: Cystic echinococcosis in the Mediterranean basin (NATO Siences Series). IOS press (Publisher); 2000; p.41-55.

21-Prousalidis J, Kosmidis Ch,Fahantidis E, Alters O: Surgical treatment of multiple cystic echinococcosis. $H P B$ (oxford). 2004; 6: 110-114.

22-Ozacmak ID, Ekiz F, Ozmen V, Isik A: Management of residual cavity after partial cystectomy for hepatic hydatosis: Comparison of omentoplasty with external drainage. Eur J Surg 2000; 166(9): 696-699.

23-Yol S, Kartal A, Sahin M, et al: Open drainage versus overlapping method in the treatment of hepatic hydatid cyst cavities. Int Surg 1999; 84 (2): 139-143.

24-Buttenschoen K, Buttenschoen D: Echinococcus granulosus infection: The challenge of surgical treatment. Langenbecks Arch Surg 2003; 388(4): 218-230. 